\title{
Thermoplastic Polyurethane Synthesis Using POSS as a Chain Modifier
}

\author{
Gabriel Hoyer Lopes ${ }^{\mathrm{a}}$, Janaína Junges ${ }^{\mathrm{a} *}$, Rudinei Fiorio $^{\mathrm{b}}$, Mara Zeni $^{\mathrm{a}}$, Ademir José Zattera ${ }^{\mathrm{a}}$ \\ ${ }^{\mathrm{a}}$ Laboratory of Polymers - LPOL, Center of Exact Sciences and Technology - CCET, \\ Caxias do Sul University - UCS, Rua Francisco Getúlio Vargas, 1130, \\ Bairro Petrópolis, CEP 95070-560, Caxias do Sul, RS, Brazil \\ ${ }^{\mathrm{b}}$ Federal Institute for Education, Science and Technology, Rua Mário de Boni, 2250, \\ Bairro Floresta, CEP 95012-580, Caxias do Sul, RS, Brazil
}

Received: September 28, 2011; Revised: May 31, 2012

\begin{abstract}
In this study, thermoplastic polyurethanes (TPUs) were synthesized using the one-shot process in solution. To obtain the samples n-phenylaminopropyl polyhedric oligomeric silsesquioxane (POSS) was added as a chain modifier during the synthesis in four different amounts. The samples were characterized by infrared spectroscopy (FTIR), thermogravimetric analysis (TGA), differential scanning calorimetry (DSC) and capillary rheometry. FTIR confirm the TPU attainment due the absence of the band at $\sim 2253 \mathrm{~cm}^{-1}$ suggesting a complete conversion of the isocyanate terminations. The TGA showed that incorporation of POSS decreased the rate of mass loss of TPU under isothermal conditions. The flexible phase showed an enhanced stability to temperature, probably due to increased phase separation between the rigid and flexible domains. The DSC showed that incorporation of $0.4 \mathrm{wt}$. (\%) of POSS increased the glass transition temperature of the flexible phase. Moreover, addition of POSS modified the melting behaviour, providing samples with a higher melting enthalpy compared to neat TPU as a consequence of the formation of larger crystals. The capillary rheometry analysis revels that the POSS addition showed a clear tendency toward higher intrinsic viscosities as the amount of POSS was increased.
\end{abstract}

Keywords: thermoplastic polyurethane, POSS, FTIR, thermal stability, DSC, capillary rheometry

\section{Introduction}

With a wide variety of applications, the chemical versatility of polyurethanes (PUs) has led to a new class of high performance materials for use as paints and varnishes, adhesives, resins, fibers, elastomers, etc. ${ }^{1,2}$.

The building blocks used to obtain PUs, both thermoplastics and thermosets, are diisocyanates and polyisocyanates, as well as compounds with a broad array of molar masses containing two or more hydroxyl groups. The isocyanate $(-\mathrm{N}=\mathrm{C}=\mathrm{O})$ and hydroxyl $(-\mathrm{OH})$ groups react forming the urethane group ${ }^{3,4}$. The thermoplastic polyurethanes (TPUs) were the first thermoplastic elastomers (TPEs) to be discovered, and one of the most important of this class of materials. The TPEs possess a different structure and morphology compared to thermoset elastomers, often lacking the usual crosslinkings created by the addition of curing agents. TPUs show distinct microphases: the flexible domains, which are generally amorphous, have a glass transition temperature $(\mathrm{Tg})$ below room temperature and are responsible for the properties being similar to those of the normal elastomers; and rigid domains, segments that act as physical crosslinks, with a relatively high $\mathrm{Tg}^{5,6}$. The rigid segment is thermally labile, allowing the material to be processed through the techniques normally applied to other thermoplastics ${ }^{4,7,8}$.

*e-mail: janainajunges@gmail.com
The TPU properties are the result from the combination of the rigid and flexible domain, consisting of coiled long polymeric chains, which are also present in the structure of conventional elastomers, the segment orientation, the amount of hydrogen bonds and other intermolecular interactions ${ }^{9}$.

As a disadvantage, the TPUs show considerable susceptibility to thermal and thermo-oxidative degradation over the temperature range required to process the material ${ }^{10}$. The urethane group has a low thermal stability, due to its reversible dissociation into isocyanate and alcohol at temperatures around $150-200{ }^{\circ} \mathrm{C}^{11,12}$.

The urethane group dissociation causes a fast decrease in the polymer molar mass, modifying the viscosity, crystallization behavior and even mechanical properties, during controlled analysis or common methods of processing in the melt state ${ }^{12}$. The thermal stability of the urethane group is dependent on the nature of the polyol chain, the chain extender and the isocyanate used. Several attempts to enhance the urethane group stability have been reported, through the addition of thermally stable isocyanates, alcohols and chain extenders ${ }^{5}$. Such modifications change the system processability, and sometimes the low temperature properties.

In this study, a polyhedric oligomeric silsesquioxane (POSS) is proposed as a stabilizing agent considering the thermal properties of the urethane group ${ }^{13,14}$. The POSS 
structure was first reported in 1946 . However, it has recently received greater attention due to its unique structure. It is formed of a cage-shaped inorganic nucleus, usually closed, comprising silicon and oxygen atoms. In monomer form, POSS molecules crystallize as spheres within a rhombohedral (or hexagonal) lattice with well-defined melting points that depend on the vertex group. Commonly, such vertex groups are linear or cyclic aliphatic molecules, engendering strong hydrophobicity to the POSS molecule. Depending on their compatibility with the polymer matrix, POSS moieties can either disperse nearly within a molecular level or aggregate into nanoscale domains ${ }^{13}$. The average size of the nucleus is $1.5 \mathrm{~nm}$, and it is surrounded by functional organic groups. The POSS nanoscopic size allows reinforcement of the polymeric segments in which it is included, controlling the chain mobility at the molecular level ${ }^{15}$. These effects are related to the surface area and chemical interactions imposed by the nanoreinforcement of the polymeric chain, and its effect is analogous to the macroscopic reinforcement obtained by the addition of fibers to a polymeric matrix in composite structures ${ }^{16,17}$. The unique characteristics of POSS make it a promising reagent in the attempt to thermally stabilize the urethane group and to evaluate the resulting thermal properties ${ }^{1}$.

In this way, the literature lacks works on the influence of POSS on the thermal stability of TPU nanocomposites. So, this work focus in evaluate the thermal stability, thermal resistance and the glass transition values of neat TPU and TPU with addition of POSS in amounts of $0.2,0.4$ and $0.6 \%$ in weight. On the other hand, FTIR was used to confirm if the synthesis process was a reaction completely effective. Also, the capillary rheometry was used to evaluated the nanocomposites rheological behaviour at higher shear rates.

\section{Material and Methods}

\subsection{Materials}

The materials used were a 4,4'-diphenylmethane diisocyanate (MDI) based pre-polymer (Urecon 185, Coim, with $18 \%$ free $-\mathrm{N}=\mathrm{C}=\mathrm{O}$ ), hydroquinone bis-(2-hydroxyethyl)ether (HQEE, Sigma-Aldrich, 98\%), n-phenylaminopropyl-polyhedric oligomeric silsesquioxane (POSS, Hybrid Plastics) and methyl ethyl ketone (MEK, Lafan Química Fina, P.A.) as a solvent. The MEK was dried using a molecular sieve. All other reagents were used as supplied.

\subsection{TPU Synthesis}

The synthesis of the polymer with POSS incorporated into the main chain was carried out in a one-shot process, in a solution of MEK, in an open system with a constant flow of nitrogen. The nitrogen was previously saturated with MEK vapor, and the system was mechanically stirred at $90 \mathrm{rpm}$. The nitrogen flow was set at $50 \mathrm{~mL} / \mathrm{min}$, and the reaction was carried out for 120 minutes. The stoichiometry was set to maintain an NCO index around $98 \%$, according to the Equation 1 below.

$$
N C O \text { Index }=\frac{E q\left[N C O\left(g^{\prime} \cdot \mathrm{mol}^{-1}\right)\right]}{E q\left[O H\left(g \cdot m o l^{-1}\right)\right]}
$$

The material obtained was removed from the reactor and transferred to a single joint glass container, connected to a spiral condenser. This system was maintained under heating for 22 hours in an oil bath for post-curing. The resulting samples were separated from the solvent through vacuum filtration and dried in a vacuum oven for 72 hours. Neat TPU and TPUs samples with $0.2,0.4$ and $0.6 \%$ by weight of POSS were obtained in triplicate.

\subsection{Attenuated total reflectance spectroscopy (ATR)}

The Fourier transform infrared spectroscopy analysis was carried on a Nicolet Impact 400 spectrometer, with a scanning range of between 400 and $4000 \mathrm{~cm}^{-1}$. The TPUs obtained were scanned in solid dust form, previously dried at $70{ }^{\circ} \mathrm{C}$ in an air circulation oven for 48 hours. Samples were compressed in order to obtain maximum contact, yielding surface spectra.

\subsection{Thermal analysis}

Thermal analysis (Shimadzu, TGA50) were carried out in triplicate under nitrogen atmosphere, with a flow rate of $50 \mathrm{~mL} / \mathrm{min}$. Samples had an approximate mass of $10 \mathrm{mg}$. The heating rate used was $10^{\circ} \mathrm{C} / \mathrm{min}$ and the temperature range was 25 to $810^{\circ} \mathrm{C}$.

Using the same equipment mentioned above, samples of around $10 \mathrm{mg}$ were quickly heated $\left(40{ }^{\circ} \mathrm{C} / \mathrm{min}\right)$ to temperatures of $210,230,260$ and $280^{\circ} \mathrm{C}$, and keep at each temperature for 60 minutes.

\subsection{Differential scanning calorimetry (DSC)}

Samples with an average mass of $10 \mathrm{mg}$ were analyzed in triplicate with a differential scanning calorimeter (DSC, Shimadzu, DSC60), contained in hermetically sealed aluminum pans. The analysis was carried under nitrogen atmosphere, with a flow rate of $50 \mathrm{~mL} / \mathrm{min}$, over a temperature range from -50 to $270{ }^{\circ} \mathrm{C}$.

\subsection{Capillary rheometry}

The capillary rheometry analysis (Galaxy III 9052, Kayeness) was carried according to the ASTM D3895-96 ${ }^{18}$, at the temperature of $230^{\circ} \mathrm{C}$, using a capillary with $\mathrm{L} / \mathrm{D}=20$. The pre-determined shear rates were approximately 100 , 200, 300, 500 and 700/s. The preset values were corrected using the Rabinowitsch correction ${ }^{19}$.

\section{Results and Discussion}

\subsection{Attenuated total reflectance spectroscopy (ATR)}

The Figure 1 showed the FTIR spectra for the neat reagents, also they chemical structure and the most important groups on MDI, HQEE and POSS reagents. The Figure 1a of the pre-polymer presents in detail a strong band at approximately $2253 \mathrm{~cm}^{-1}$ assigned to NCO groups ${ }^{1,13}$. Figure $1 \mathrm{~b}$ showed the prominent band at about $3500-3000 \mathrm{~cm}^{-1}$ assigned to $\mathrm{OH}$ groups, and $\mathrm{CH}_{2}$ and $\mathrm{CH}_{3}$ stretching bands at 2930-2869 $\mathrm{cm}^{-1}$ characteristic of the HQEE structure ${ }^{20}$. Figure $1 \mathrm{c}$ shows the band at $3300 \mathrm{~cm}^{-1}$ 
ascribed to $\mathrm{NH}$ groups and stretching of $\mathrm{CH}_{(\mathrm{Ar})}$ from aromatic groups in $3040 \mathrm{~cm}^{-1}$ both assigned to n-phenylaminopropyl radical structure. Also, the characteristics POSS bands Si-O and $\mathrm{Si}-\mathrm{O}-\mathrm{R}$ appears in the range between $1400-1000 \mathrm{~cm}^{-1[13]}$.

The Figure 2 presents the four samples synthesized. Initially the spectra of the four samples in Figure 2 were compared with the reagents spectra in Figure 1. The different TPUs, regardless of the amount of POSS added to the

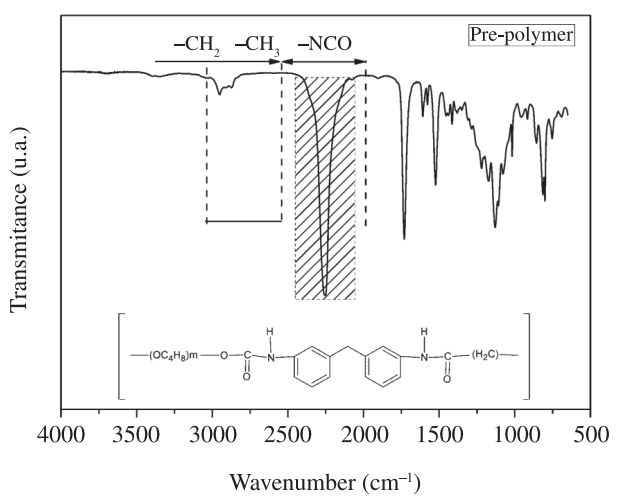

(a)

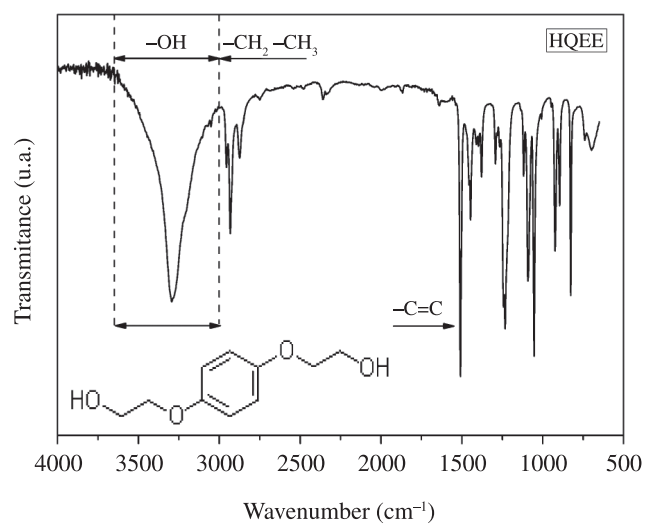

(b)

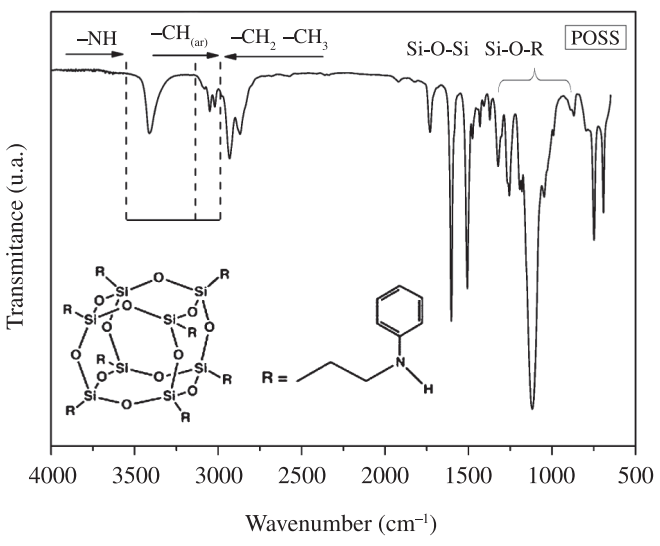

(c)

Figure 1. FTIR spectra of TPU reagents: a) pre-polymer; b) HQEE and; c) POSS. formulation, showed very similar spectra. The Figure 2 showed the prominent band at about 3400-3200 $\mathrm{cm}^{-1}$ assigned to $\mathrm{OH}$ groups. As an expected consequence, unreacted hydroxyl and secondary amines, as well as the $\mathrm{N}-\mathrm{H}$ group present in the urethane and urea, were detected as a single peak in the same region. As the characteristic peaks of each above-mentioned group are all present in the same general region, the spectra shows a single peak with medium intensity and a large area. The aforementioned groups are present at the polymer chain ends, and at the end of the organic functions attached to the POSS nuclei ${ }^{20,21}$. The absorption peak observed between $3043-3041 \mathrm{~cm}^{-1}$ is associated with the presence of aromatic rings in the MDI, HQEE and organic terminations of the POSS (Figure 1). Peaks immediately below the $3000 \mathrm{~cm}^{-1}$ region show the presence of $\mathrm{C}-\mathrm{H}$ bonds, confirmed in the region of $1455-1414 \mathrm{~cm}^{-1[15]}$.

The characteristic $\mathrm{N}=\mathrm{C}=\mathrm{O}$ peak at approximately $2253 \mathrm{~cm}^{-1}$ was absent in Figure 2, suggesting a complete conversion of the isocyanate terminations presents on MDI reagent in Figure 1a into urethane and urea groups. This is an indicative of completely reaction as showed on the detail region in Figure 1a and Figure $2^{13}$. Because of the excellent compatibility of POSS in TPU, when the POSS content was overdue, all the NCO groups would react with POSS ${ }^{1}$.

The absorption at $1732-1703 \mathrm{~cm}^{-1}$ is associated with the $\mathrm{C}=\mathrm{O}$ groups in free urethanes ${ }^{20}$, which are present in the urethane groups, and also ester groups present in the polyol chains of the pre-polymer. Absorption at $1595 \mathrm{~cm}^{-1}$ is also characteristic of the above-mentioned aromatic groups. Further evidence of the presence of $\mathrm{N}-\mathrm{H}$ groups is detected through peaks in the $1532-1508 \mathrm{~cm}^{-1}$ region, consistent with the expected formation of urethane groups and the presence of associated secondary amines from the organic groups attached to the POSS nuclei ${ }^{20,21}$. Peaks situated at $1310-1308 \mathrm{~cm}^{-1}$ correspond to the $\mathrm{C}-\mathrm{O}$ group present in esters and those in the range of $1238-1223 \mathrm{~cm}^{-1}$ are related to three different groups, these being aliphatic $\mathrm{C}-\mathrm{N}$, aromatic $\mathrm{C}-\mathrm{N}$ and $\mathrm{C}-\mathrm{O}$ in ethers. As all three groups are present in the formulation, overlapping of the above-mentioned peaks is likely to occur ${ }^{20}$.

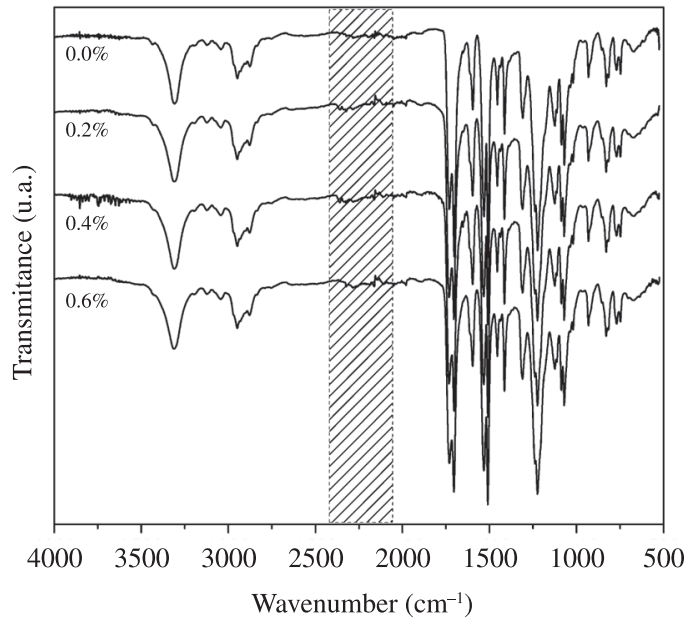

Figure 2. FTIR spectra of synthesized TPU using different amounts of POSS. 
The $\mathrm{Si}-\mathrm{O}$ and $\mathrm{Si}-\mathrm{O}-\mathrm{R}$ groups, with a characteristic peak in the $1100-1000 \mathrm{~cm}^{-1}$ region ${ }^{13,21-22}$, cannot be distinguished from the esther groups detected at $1124-1070 \mathrm{~cm}^{-1}$. Such groups are present in the polyol component of the pre-polymer, and the small amount of POSS in the formulation probably cannot be detected due to overlapping of the ether group peaks. Observation of the absorption characteristics below $1000 \mathrm{~cm}^{-1}$ is used to characterize the position of substitution of the aromatic rings dispersed in the polymer chain of the synthesized TPU ${ }^{20}$, where at $\sim 829 \mathrm{~cm}^{-1}$ the p-substituted aromatic rings, present from the MDI and HQEE, are detected; and the peaks at 770-747 $\mathrm{cm}^{-1}$ are characteristic of the mono-substituted aromatic rings of the n-phenyl groups of the POSS ${ }^{21}$. Based on the FTIR results, the development synthesized process shows to be an attractive method to obtain TPU/POSS nanocomposites without traces of unreacted NCO groups.

\subsection{Thermal stability}

For the synthesized TPUs, samples decomposed mainly in two steps. The first step could be attributed to the degradation of the hard or rigid domains of the TPU, and is commonly related to urethane break bonds, occurring between $250-350{ }^{\circ} \mathrm{C}$. The second step could be related to the degradation of the elastic or flexible segments, and this event is situated in the range of $350-500{ }^{\circ} \mathrm{C}^{1,23}$. By increasing the POSS content, a shift to higher temperatures, for the first and the second range of degradation, can be noted in Table 1 despite the relatively small amount of POSS added.

According to Liu et al. ${ }^{24}$, the higher thermal stability of the TPU/POSS nanocomposites may be associated with an arrangement of interconnected structures involving the polymer matrix and POSS cages, promotes a significant effect on the TPU rigid domain. As showed in the Table 1, the peak temperature increases from $333.9 \pm 0.1$ to $352.1 \pm 1.8$. The increase in the thermal stability is related also to the barrier effect caused by the formation of inert silicate layers that can act as a heat transfer barrier, since POSS is an inorganic material and has lower thermal conductivity than the regular carbon segments ${ }^{25}$. In addition POSS tends to crystallize in two dimensions in a format of lamellar structures ${ }^{26}$.

There are significant changes in the on set and peak temperatures of the flexible domain, as well. As showed in Table 1, the peak temperatures for TPU/POSS samples improve approximately $13{ }^{\circ} \mathrm{C}$ when compared with neat TPU. These results may be due to the presence of urea groups, formed by the reaction with the secondary amine

Table 1. Thermal stability of the synthesized TPUs.

\begin{tabular}{ccccc}
\hline $\begin{array}{c}\text { POSS } \\
\text { Fraction } \\
(\boldsymbol{\%})\end{array}$ & $\begin{array}{c}\text { Onset }_{\text {d-r }} \\
\left({ }^{\circ} \mathbf{C}\right)\end{array}$ & $\begin{array}{c}\mathbf{T}_{\text {d-r }} \\
\left({ }^{\circ} \mathbf{C}\right)\end{array}$ & $\begin{array}{c}\text { Onset }_{\text {d-f }} \\
\left({ }^{\circ} \mathbf{C}\right)\end{array}$ & $\begin{array}{c}\mathbf{T}_{\text {d-f }} \\
\left({ }^{\circ} \mathbf{C}\right)\end{array}$ \\
\hline 0.0 & $295.4 \pm 2.3$ & $333.9 \pm 0.1$ & $367.2 \pm 2.4$ & $375.6 \pm 0.8$ \\
0.2 & $296.4 \pm 0.6$ & $352.1 \pm 1.8$ & $381.1 \pm 1.7$ & $392.6 \pm 3.0$ \\
0.4 & $299.6 \pm 1.3$ & $348.2 \pm 0.8$ & $378.1 \pm 0.3$ & $392.3 \pm 0.1$ \\
0.6 & $298.5 \pm 2.5$ & $348.3 \pm 0.7$ & $379.0 \pm 1.2$ & $404.4 \pm 2.4$ \\
\hline
\end{tabular}

Notes: Subscribed 'f' refers to the flexible domain and $r$ to the rigid domain in the polymer. group attached to the POSS nuclei. The urea group, as well as the urethane group and the POSS, are highly polar, and thus it is difficult to separate each exact degradation temperature due to possible overlapping of each degradation phenomenon. The POSS can also induce a higher phase separation $^{21}$, causing changes in the degradation mechanism of the flexible domain. These improvements are associated with the increase in the POSS ramification flexibility as well a reduction in the average crosslink density ${ }^{24,27-28}$. Also the resulting properties are dependent on the reactivity of the functional groups present in the nanocages ${ }^{29}$.

\subsection{Thermal resistance}

According to the data obtained from the curves (Figure 3 and Table 2), the measured mass losses were slightly lower in the TPUs with POSS additions. The $0.4 \%$ POSS presented the lowest mass loss at all temperatures, except at $260{ }^{\circ} \mathrm{C}$, probably due to a lower molar mass in that specific sample. Further addition of POSS yielded polymers with slightly higher mass loss. The change in behavior can be explained by the addition of urea groups, through the reaction of isocyanates with the secondary amine groups present in the POSS. Urea groups are more stable than urethane groups, leading to an increase in thermal resistance ${ }^{20}$.

These could be attributed to the consolidation effects which could be interpreted on the following two factors.

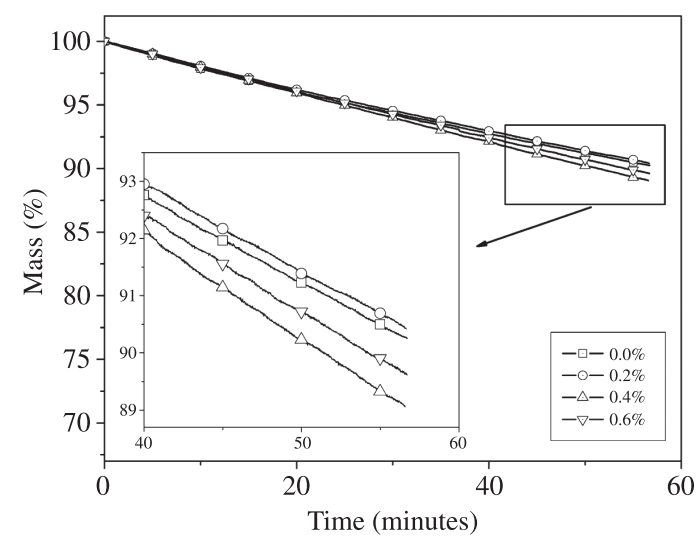

(a)

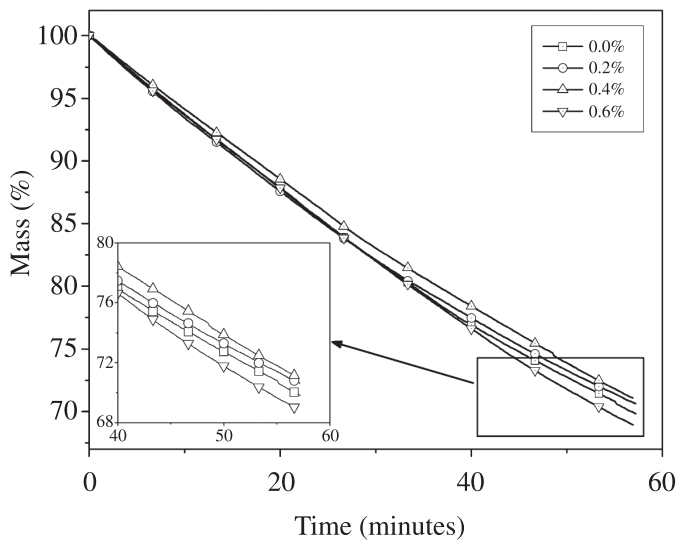

(b)

Figure 3. TGA curves of TPUs at (a) at $260{ }^{\circ} \mathrm{C}$ and; (b) at $280{ }^{\circ} \mathrm{C}$. 
(1) The TPU chain movement may was restricted by the increasing crosslinking density of materials as POSS content increased. (2) When POSS content was low, TPU/POSS nanocomposites exhibited rubberlike properties because of the main part of the composite was soft segments ${ }^{1}$.

\subsection{Differential scanning calorimetry (DSC)}

The DSC heating traces for all of our samples, while glass transition temperatures, melting points, and latent heats of melting are summarized in Table 3 . The average melting temperature (Tm) was significantly higher for the obtained TPUs, comparing polymers using 1,4-buthanediol (BDO) as a short chain extender ${ }^{23,30}$. Comparing the different formulations, melting temperatures does not show significant changes with the addition of POSS. According to the Flory-Huggins theory ${ }^{31}$, a melting temperature increase indicates a comparably unfavorable interaction between components, in this case, the TPU and POSS. Considering this theory, the addition of POSS drives stronger phase separation of the TPU hard segment from the amorphous phase in POSS TPUs, leading to enhanced POSS crystallization ${ }^{15}$.

The melting enthalpy showed appreciable changes only for TPUs with $0.4 \%$ addition of POSS; the glass transition temperature of flexible phase $\left(\mathrm{T}_{\mathrm{g}-\mathrm{f}}\right)$ values consequently being higher due to the lower chain mobility. These findings indicate changes in the amount of crystal and the size of such structures sizes, where even relatively small amounts of POSS interfere with the polymer chain interactions. The non-linear behavior may be due to crosslinking occurring in the TPU during the synthesis, which can be induced by the presence of moisture remaining in the system, as well as the amount of functional groups attached to the POSS nuclei (eight secondary amine groups per POSS molecule).

$\mathrm{Xu}$ et al..$^{32,33}$ investigated the Tg enhancement mechanism of POSS-based hybrid polymers employing FTIR spectra and reported that a strong interaction between POSS siloxane and the polar carbonyl group contributes to a

Table 2. TPU mass loss as a function of temperature.

\begin{tabular}{ccccc}
\hline \multirow{2}{*}{$\%$ POSS } & \multicolumn{4}{c}{ Mass loss $(\boldsymbol{\%})$} \\
\cline { 2 - 5 } & $\mathbf{2 1 0}{ }^{\circ} \mathbf{C}$ & $\mathbf{2 3 0}^{\circ} \mathbf{C}$ & $\mathbf{2 6 0}^{\circ} \mathbf{C}$ & $\mathbf{2 8 0}^{\circ} \mathbf{C}$ \\
\hline 0.0 & $1.6 \pm 0.3$ & $2.2 \pm 0.2$ & $9.4 \pm 0.3$ & $28.6 \pm 0.6$ \\
0.2 & $1.4 \pm 0.1$ & $1.5 \pm 0.2$ & $9.2 \pm 0.3$ & $28.0 \pm 0.3$ \\
0.4 & $1.2 \pm 0.1$ & $1.5 \pm 0.1$ & $10.6 \pm 0.6$ & $27.6 \pm 0.7$ \\
0.6 & $1.5 \pm 0.2$ & $1.8 \pm 0.1$ & $10.1 \pm 0.4$ & $29.8 \pm 0.3$ \\
\hline
\end{tabular}

Table 3. DSC data for the neat TPU and TPU/POSS samples.

\begin{tabular}{ccccr}
\hline $\begin{array}{c}\text { POSS } \\
\text { fraction } \\
(\%)\end{array}$ & $\mathbf{T}_{\mathbf{m}}\left({ }^{\circ} \mathbf{C}\right)$ & $\Delta \mathbf{H}_{\mathbf{m}}(\mathbf{J} . \mathbf{g}-\mathbf{1})$ & $\mathbf{T}_{\mathbf{g}-\mathbf{f}}\left({ }^{\circ} \mathbf{C}\right)$ & $\mathbf{T}_{\mathbf{g}-\mathbf{r}}\left({ }^{\circ} \mathbf{C}\right)$ \\
\hline 0.0 & $237.7 \pm 2.8$ & $-37.8 \pm 0.4$ & $-13.4 \pm 0.6$ & $97.7 \pm 1.7$ \\
0.2 & $244.1 \pm 2.5$ & $-37.8 \pm 3.5$ & $-17.6 \pm 3.1$ & $102.4 \pm 0.5$ \\
0.4 & $244.2 \pm 0.3$ & $-44.4 \pm 1.2$ & $-12.7 \pm 1.2$ & $98.9 \pm 3.1$ \\
0.6 & $243.0 \pm 2.8$ & $-33.8 \pm 1.2$ & $-21.2 \pm 3.8$ & $102.0 \pm 0.5$ \\
\hline
\end{tabular}

Notes: Subscribed 'f' refers to the flexible domain and " $r$ " to the rigid domain in the polymer. significant $\mathrm{Tg}$ increase. In good agreement with their observations, we also found that, for instance, the FTIR spectrum of TPU/POSS exhibited peaks at $1310-1070 \mathrm{~cm}^{-1}$ (Figure 2). In our case, the polar groups, which induce the dipole-dipole interaction with POSS siloxane, could be the carbonyl group and/or ether group of the TPU backbone. Because of the microphase-separated microstructure, this dipole-dipole interaction might mainly take place at the interface between the POSS and the TPU. This increase in Tg with POSS loading, despite the microphase-separated microstructure, indicates an interfacial effect where in the rigid POSS phase decreases segmental flexibility of the adjacent TPU phase, leading to the Tg enhancement ${ }^{13}$. The exception of $0.4 \%$ POSS may be due to the high crystallinity of TPU domains for that sample. In addition, as POSS loadings increased, the crosslinking density of TPU/POSS network increased and the free volumes of the network decreased rapidly, thus the $\mathrm{Tg}$ of the network increased ${ }^{1}$.

\subsection{Capillary rheometry}

While rheology is not the focus of the present paper, it is well know that changes in the thermal behavior reflect in rheological properties of dispersions systems such as the present TPU/POSS ${ }^{34}$. The addition of POSS showed a clear tendency toward higher intrinsic viscosities as the amount of the above-mentioned chain modifier was increased. Samples tended toward a linear pseudoplastic behaviour, with a higher amount of POSS leading to higher intrinsic viscosities at all shear rates evaluated. The incorporation of POSS into the TPU backbone produced a significant change in the viscosity. The viscosity increases linearly with POSS, corroborating the reinforcing efficiency of POSS in the TPU backbone. The viscosity strongly depends on frequency (Figure 4), revealing the non-Newtonian behavior of TPU/POSS composites in which the viscosity at low frequencies is significantly higher than that obtained at high frequencies ${ }^{34}$.

The POSS restraint the movement of flexible domain probably by the formation of silicate layers, and as consequence the viscosity increase. Similar behaviour was also found by Nanda et al. ${ }^{34}$ in Polyurethane/POSS Hybrid

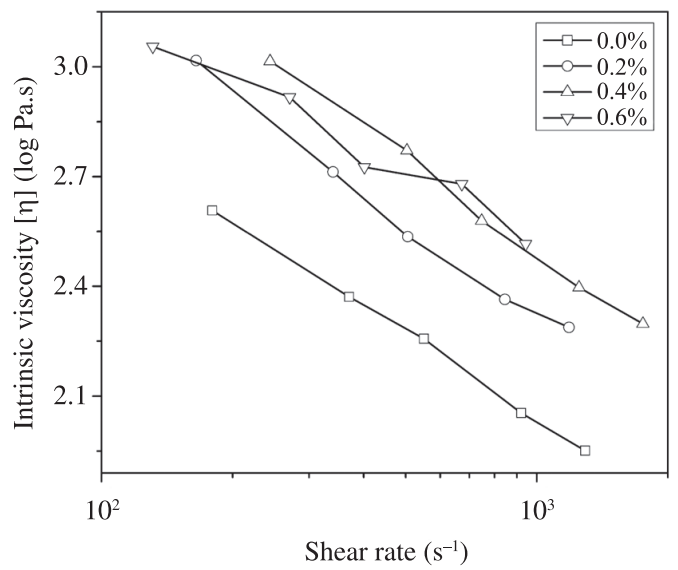

Figure 4. Intrinsic viscosity as a function of shear rate for the studied TPUs. 
system. On the other hand, the inert silicate layers may act as a heat transfer barrier, and the thermal properties can also improved $^{24,25}$, as can be seen in Table 1 and Table 2. Other functional groups could be formed from the isocyanate, such as the isocyanurate, which have a higher thermal stability than urethane groups ${ }^{23}$. However, such groups can lead to the formation of ramifications and possible crosslinking in the polymer chain. The DSC analysis did not show evidence of this behaviour, considering that the Tm remained roughly the same for all samples, and no significant decrease in the melting enthalpy was observed, although some degree of crosslinking and ramification would be expected, due to the amount of functional groups attached to the POSS nuclei.

\section{Conclusions}

TPUs were successfully synthesized from the proposed reagents, with an apparent incorporation of POSS in the main polymer chain due the absent of the band at $2253 \mathrm{~cm}^{-1}$ assigned to NCO groups. Based on the FTIR results, the development synthesized process shows to be an attractive method to obtain TPU/POSS nanocomposites.

The insertion of POSS on TPU backbone increased the thermal stability of the rigid and flexible domain. The thermal resistance also improved with POSS addition, resulting in comparatively lower mass loss during isothermal

\section{References}

1. Zhang Q, He H, Xi K, Huang X, Yu X and Jia X. Synthesis of $\mathrm{N}$-Phenylaminomethyl POSS and Its Utilization in Polyurethane. Macromolecules. 2011; 44:550-557. http:// dx.doi.org/10.1021/ma101825j

2. Holden G, Legge NR, Quirk R and Schroeder HE. Thermoplastic Elastomers. Munich: Hanser; 1996.

3. Lu QW, Hernandez-Hernandez ME and Macosko CW. Explaining the abnormally high flow activation energy of thermoplastic polyurethanes. Polymer. 2003; 44:3309-3318. http://dx.doi.org/10.1016/S0032-3861(03)00223-4

4. Pompe G, Pohlers A, Pötsche P and Piontek J. Influence of processing conditions on the multiphase structure of segmented polyurethane. Polymer. 1998; 39:5147-5153. http://dx.doi. org/10.1016/S0032-3861(97)10350-0

5. Oertel G. Polyurethane Handbook. New York: Hanser; 1993.

6. Howard GT. Biodegradation of polyurethane: a review. International Biodeterioration \& Biodegradation. 2002; 49:245-252. http://dx.doi.org/10.1016/ S0964-8305(02)00051-3

7. Lu QW and Macosko CW. Comparing the compatibility of various functionalized polypropylenes with thermoplastic polyurethane (TPU). Polymer. 2004; 45:1981-1991. http:// dx.doi.org/10.1016/j.polymer.2003.12.077

8. Li S, Tang X, Luo Y and Xu X. The study of a thermoplastic polyurethane ionomer system. European Polymer Journal. 1998; 34:1899-1902. http://dx.doi.org/10.1016/ S0014-3057(98)00027-5

9. Yeganeh $\mathrm{H}$ and Shamekhi MA. Poly(urethane-imide-imide), a new generation of thermoplastic polyurethane elastomers with enhanced thermal stability. Polymer. 2004; 45:359-365. http:// dx.doi.org/10.1016/j.polymer.2003.11.006
TGA. The improve in both thermal properties may be related to the barrier effect caused by the formation of inert silicate layers that can act as a heat transfer barriers, in addition POSS is an inorganic material and has lower thermal conductivity than the regular carbon segments from the polymer matrix. On the other hand, the DSC analysis showed an increase in $\mathrm{Tg}$ of flexible and rigid domains with POSS loading, has a result of restricts imposed to the movement of TPU chain segments. Moreover, the viscosity also increase due the restricts imposed by POSS to polymer segments.

In general, the modifications in the thermal properties of TPUs are probably influenced by an increased phase separation imposed by the volume of the POSS nuclei, formation of inert silicate layers, restriction of the chain mobility due the POSS addition, as well as the degree of crosslinking, and probably by the presence of more thermally stable urea groups.

\section{Acknowledgments}

The authors would like to thank the National Council of Technological and Scientific Development (CNPq), the Research Funding Foundation of Rio Grande do Sul (FAPERGS) for financing the project, and the University of Caxias do Sul (UCS).

10. Finnigan B, Martin D, Halley P, Truss R and Campbell K. Morphology and properties of thermoplastic polyurethane nanocomposites incorporating hydrophilic layered silicates. Polymer. 2004; 45:2249-2260. http://dx.doi.org/10.1016/j. polymer.2004.01.049

11. Nair PR, Nair CPR and Francis DJ. Phosphazene-modified polyurethanes: Synthesis, mechanical and thermal characteristics. European Polymer Journal, 1996; 32:1415-1420. http://dx.doi. org/10.1016/S0014-3057(96)00079-1

12. Hentschell $\mathrm{T}$ and Münsted H. Kinetics of the molar mass decrease in a polyurethane melt: a rheological study. Polymer. 2001; 42:3195-3203. http://dx.doi.org/10.1016/ S0032-3861(00)00489-4

13. Wu J, Ge Q and Mather PT. PEG-POSS Multiblock Polyurethanes: Synthesis, Characterization, and Hydrogel Formation. Macromolecules. 2010; 43:7637-7649. http:// dx.doi.org/10.1021/ma101336c

14. Janowski B and Pielichowski K. Thermo(oxidative) stability of novel polyurethane/POSS nanohybrid elastomers. Thermochimica Acta. 2008; 478:51-53. http://dx.doi. org/10.1016/j.tca.2008.08.015

15. Guo Q, Knight PT, Wu J and Mather PT. Blends of Paclitaxel with POSS-Based Biodegradable Polyurethanes: Morphology, Miscibility, and Specific Interactions. Macromolecules. 2010; 43:4991-4999. http://dx.doi. org/10.1021/ma100662x

16. Bourbigot S, Turf T, Bellayer $\mathrm{S}$ and Duquesne $\mathrm{S}$. Polyhedral oligomeric silsesquioxane as flame retardant for thermoplastic polyurethane. Polymer Degradation and Stability. 2009; 94:1230-1237. http://dx.doi.org/10.1016/j. polymdegradstab.2009.04.016

17. Seymour RW and Cooper SL. Thermal Analysis of Polyurethane Block Polymers. Macromolecules. 1973; 6:48-53. http://dx.doi. org/10.1021/ma60031a008 
18. American Society for Testing and Materials - ASTM. ASTM D3895: Standart test method for determination of properties of polymeric materials by means of a capillary rheometer. ASTM; 1996.

19. Bretas RES and D’Avila MA. Melts Polymer Rheology. São Carlos: EdUFSCar; 2000.

20. Silverstein M and Webster FX. Spectrometric identification of organic compunds. Rio de Janeiro: Guanabara; 1979.

21. Chattopadhyay DK and Webster DC. Thermal stability and flame retardancy of polyurethanes. Progress in Polymer Science. 2009; 34:1068-1133. http://dx.doi.org/10.1016/j. progpolymsci.2009.06.002

22. Schuur M, Noordover B and Gaymans RJ. Polyurethane elastomers with amide chain extenders of uniform length. Polymer. 2006; 47:1091-1100. http://dx.doi.org/10.1016/j. polymer.2005.11.074

23. Herrera M, Matuschek G and Kettrup A. Thermal degradation of thermoplastic polyurethane elastomers (TPU) based on MDI. Polymer Degradation and Stability. 2002; 78:323-331. http:// dx.doi.org/10.1016/S0141-3910(02)00181-7

24. Liu Y, Zheng S and Nie K. Epoxy nanocomposites with octa(propylglycidyl ether) polyhedral oligomeric silsesquioxane. Polymer. 2005; 46:12016-12025. http://dx.doi. org/10.1016/j.polymer.2005.09.056

25. Zhang Z, Gu A, Liang G, Ren P, Xie J and Wang X. Thermo-oxygen degradation mechanisms of POSS/ epoxy nanocomposites. Polymer Degradation and Stability. 2007; 92:1986-1993. http://dx.doi.org/10.1016/j. polymdegradstab.2007.08.004

26. Zheng L, Hong S and Cardoen G. Polymer Nanocomposites through Controlled Self-Assembly of Cubic Silsesquioxane Scaffolds. Macromolecules. 2004; 37:8606-8611. http://dx.doi. org $/ 10.1021 / \mathrm{ma} 048557 \mathrm{c}$

27. Pellice SA, Fasce DP and Williams RJJ. Properties of epoxy networks derived from the reaction of diglycidyl ether of bisphenol A with polyhedral oligomeric silsesquioxanes bearing $\mathrm{OH}$-functionalized organic substituents. Polymer Physics. 2003; 41:1451-1461. http://dx.doi.org/10.1002/ polb. 10494

28. Ni Y, Zhang S and Nie K. Morphology and thermal properties of inorganic-organic hybrids involving epoxy resin and polyhedral oligomeric silsesquioxanes. Polymer. 2004; 45:5557-5568. http://dx.doi.org/10.1016/j.polymer.2004.06.008

29. Strachota A, Whelan P, Krı́z J, Brus J, Urbanova M, Slouf $\mathrm{M}$ et al. Formation of nanostructured epoxy networks containing polyhedral oligomeric silsesquioxane (POSS) blocks. Polymer. 2007; 48:3041-3058. http://dx.doi.org/10.1016/j. polymer.2007.03.052

30. Koberstein JT and Galambos AF. Multiple melting in segmented polyurethane block copolymers. Macromolecules. 1992; 25:5618-5624. http://dx.doi. org/10.1021/ma00047a010

31. Flory PJ. Principles of Polymer. New York: Cornell University Press; 1953.

32. Xu HY, Kuo SW, Lee JS and Chang FC. Preparations, Thermal Properties, and Tg Increase Mechanism of Inorganic/ Organic Hybrid Polymers Based on Polyhedral Oligomeric Silsesquioxanes. Macromolecules. 2002 35:8788-8793. http:// dx.doi.org/10.1021/ma0202843

33. Xu HB, Yang BH, Wang JF, Guang SY and Li C. Preparation, Thermal Properties, and $\mathrm{Tg}$ Increase Mechanism of Poly(acetoxystyrene-co-octavinyl-polyhedral oligomeric silsesquioxane) Hybrid Nanocomposites. Macromolecules. 2005; 35:10455-10460. http://dx.doi. org/10.1021/ma0516687

34. Nanda AK, Wicks DA, Madbouly AS and Otaigbe JU. Nanostructured Polyurethane/POSS Hybrid Aqueous Dispersions Prepared by Homogeneous Solution Polymerization. Macromolecules. 2006; 39:7037-7043. http:// dx.doi.org/10.1021/ma060809h 